\title{
Clinical Significance of Serum Carcinoembryonic Antigen Level in Rectal Cancer Patients Who Underwent Preoperative Chemoradiotherapy
}

\author{
Jin Cheon Kim \\ Department of Surgery, Asan Medical Center, University of Ulsan College of Medicine, Seoul, Korea
}

\section{See Article on Page 205-212}

Complete or near-complete pathologic response after preoperative chemoradiotherapy (CRT) for the treatment of locally advanced rectal cancer (LARC) is known to reduce local recurrence, to increase the survival rate, and concurrently to enable sphincter-preserving operations $[1,2]$. Preoperative CRT has, therefore, become a standard component of combined treatment for LARC, namely, T3-4 or N+ rectal cancers. In particular, the subgroup of patients who achieve a pathologically complete response (pCR) has a very low risk of local or distant recurrence. Several prognostic parameters, radiological findings, and tumor markers have been suggested to correlate with the response to treatment in rectal cancer, but their clinical usefulness to predict CRT responses in an accurate or timely manner remains controversial. Recently, serum carcinoembryonic antigen (s-CEA) has been investigated as a prognostic marker in patients who have undergone preoperative CRT.

The role of s-CEA in determining the prognosis after a radical resection for LARC has been well documented. Perez et al. [3] reported that post-CRT s-CEA levels lesser than $5 \mathrm{ng} / \mathrm{mL}$ were associated with an increased rate of downstaging, with pCR, and with a favorable prognosis [4]. In our recent study involving 333 LARC patients who underwent preoperative CRT, CEA grouping according to changes in the s-CEA concentration by using the ratio of pre- to post-CRT (70\% as a cut-off value) presented an independent prognostic factor for disease-free survival with-

Correspondence to: Jin Cheon Kim, M.D.

Department of Surgery, Asan Medical Center, University of Ulsan College of Medicine, 88 Olympic-ro 43-gil, Songpa-gu, Seoul 138-736, Korea

Tel: +82-2-3010-3489, Fax: +82-2-474-9027

E-mail: jckim@amc.seoul.kr out showing any correlation between pre-CRT s-CEA level and pathologic tumor response [5]. Presently, few biomarkers are considered promising as CRT-predictive markers. The s-CEA level of pre- or post-CRT appears to be closely associated with the prognosis in LARC patients, but its predictive value for pathologic response needs to be further verified. Additionally, efficient individualized regimens must be continuously investigated for patients with poor CRT-response and/or elevated s-CEA after CRT.

\section{REFERENCES}

1. Roh MS, Colangelo LH, O'Connell MJ, Yothers G, Deutsch M, Allegra CJ, et al. Preoperative multimodality therapy improves disease-free survival in patients with carcinoma of the rectum: NSABP R-03. J Clin Oncol 2009;27:5124-30.

2. Park JH, Yoon SM, Yu CS, Kim JH, Kim TW, Kim JC. Randomized phase 3 trial comparing preoperative and postoperative chemoradiotherapy with capecitabine for locally advanced rectal cancer. Cancer 2011;117:3703-12.

3. Perez RO, Sao Juliao GP, Habr-Gama A, Kiss D, Proscurshim I, Campos FG, et al. The role of carcinoembriogenic antigen in predicting response and survival to neoadjuvant chemoradiotherapy for distal rectal cancer. Dis Colon Rectum 2009;52:1137-43.

4. Yoon SM, Kim DY, Kim TH, Jung KH, Chang HJ, Koom WS, et al. Clinical parameters predicting pathologic tumor response after preoperative chemoradiotherapy for rectal cancer. Int J Radiat Oncol Biol Phys 2007;69:1167-72.

5. Kim CW, Yu CS, Yang SS, Kim KH, Yoon YS, Yoon SN, et al. Clinical significance of pre- to post-chemoradiotherapy s-CEA reduction ratio in rectal cancer patients treated with preoperative chemoradiotherapy and curative resection. Ann Surg Oncol 2011;18: 3271-7. 